\title{
Relação entre achados ultrassonográficos de tendinopatia e bursopatia de ombro e incapacidade para o trabalho
}

\section{Relationship between tendinopathy and bursopathy findings in ultrasonography of the shoulder and work disability}

\author{
Fernando Pessoa Weiss' ${ }^{1}$; Talita Zerbini²
}

\begin{abstract}
Weiss FP; Zerbini T. Relação entre achados ultrassonográficos de tendinopatia e bursopatia de ombro e incapacidade para o trabalho. Saúde, Ética \& Justiça. 2013;18(2):157-66.

RESUMO: Achados como tendinopatia e bursopatia são comumente encontrados em exames de ultrassonografia de ombro, mas nem sempre geram repercussão clínica. Consequentemente, tais achados não são interpretados pelo médico perito como doença ou lesão. Isso acaba gerando muitas contestações por procuradores e juízes a respeito da qualidade técnica do laudo pericial. O presente estudo tem por objetivo avaliar a interpretação dos médicos peritos quanto à relação entre os achados de tendinopatia e bursopatia em exames de ultrassonografia do ombro e a incapacidade para o trabalho. Para isso, foram analisados os laudos periciais entre 01.08.2013 e 01.12.2013 do Juizado Especial Federal Previdenciário de Curitiba nos quais o avaliado tinha como queixa principal a dor no ombro, e que os achados ultrassonográficos fossem condizentes com objetivo do estudo. Os resultados foram avaliados considerando os sinais clínicos encontrados, os achados dos exames ultrassonográficos e a determinação de incapacidade para o trabalho. Dentre os 21 indivíduos incluídos no trabalho, houve correspondência entre as alterações ultrassonográficas e positividade dos testes clínicos em $42,8 \%$ dos casos. No entanto, destes, apenas $33 \%$ foram definidos com portadores de incapacidade para o trabalho. Com base neste estudo concluiu-se que apenas $14,3 \%$ dos indivíduos que apresentavam as alterações ultrassonográficas estudadas foram considerados incapazes para o trabalho, o que evidencia que somente achados isolados de exames complementares não são determinantes para a avaliação da capacidade laboral.
\end{abstract}

DESCRITORES: Bainha rotadora; Ultrassonografia; Avaliação de desempenho profissional.

\footnotetext{
1. Médico Ortopedista, Pós-graduando do Curso de Especialização em Perícias Médicas e Medicina Legal da Faculdade de Ciências Médicas da Santa Casa de São Paulo.

2. Professora colaboradora da Especialização em Perícias Médicas e Medicina Legal da Faculdade de Ciências Médicas da Santa Casa de São Paulo.

Endereço para correspondência: Autor correspondente: Fernando Pessoa Weiss. Rua Nunes Machado, 1614, Curitiba, PR, CEP 80220-070. E-mail: fernandoweiss@docpericias.com.bre fpweiss@hotmail.com
} 


\section{INTRODUÇÃO}

$\mathbf{N}$

a prática diária do ortopedista generalista, aproximadamente $10 \%$ de seus pacientes apresentam queixas envolvendo o ombro. Destes casos, a metade é de queixas referentes especificamente ao manguito rotador ${ }^{1}$.

A dificuldade de diagnóstico sindrômico e etiológico pelo médico assistente e de correlação dos achados clínicos com os achados de exames de imagem, especialmente em exames de ultrassonografia do ombro, tornam a perícia médica um grande desafio para o perito médico, que necessita utilizar vasto arsenal propedêutico a fim de sanar, não só as dúvidas referentes ao real diagnóstico, mas especialmente aquelas relacionadas à capacidade do avaliado para o trabalho.

A articulação do ombro é composta por quatro articulações: glenoumeral, acromioclavicular, esternoclavicular e escapulotorácica, que trabalham em conjunto para promover a harmonia durante os movimentos do ombro ${ }^{2}$.

Dentre as estruturas que compõem e estabilizam o ombro, damos uma atenção especial aos músculos que compõem o manguito rotador, são eles: o tendão supraespinal, o redondo menor, o infraespinal e o subescapular. Os três primeiros com função primária de rotação externa, o último com função de rotação interna ${ }^{3}$.

Em 1937, Codman propôs uma maior susceptibilidade do tendão supraespinal a lesões devido à baixa vascularização encontrada a aproximadamente 01 $\mathrm{cm}$ da grande tuberosidade, região que ficou popularmente conhecida como zona crítica de Codman ${ }^{4}$.

$\mathrm{Na}$ década de 70, estudos de Neer nos apresentaram o conceito de síndrome de impacto, definido pela hipótese de os tendões do manguito rotador sofrerem impacto contra estruturas osteoligamentares do arco coracoacromial do ombro.

Com base nos estudos de Neer, foi proposta a classificação evolutiva da lesão dos tendões do manguito rotador: estágio I: edema e hemorragia na bursa, habitualmente em pessoas jovens, abaixo de 25 anos; estágio II: fibrose e tendinite, habitualmente na faixa entre 25-45 anos; estágio III: alterações crônicas, que envolvem lesão de fibras de forma parcial ou total do tendão (Neer, 1983) $)^{5}$.

Portanto, qualquer estrutura que fique interposta entre a cabeça do úmero e o arco coracoacromial pode sofrer impacto, seja os tendões do manguito, a bursa subacromial ou a região proximal do cabo longo do bíceps, o que caracteriza a síndrome de impacto ${ }^{2}$.

A etiologia desta síndrome é multifatorial, isto é, causas intrínsecas (intratendão) e extrísecas (extratendão) estão envolvidas no complexo mecanismo de impacto. Dentre os fatores intrínsecos destacam-se alterações neurológicas que cursem com desequilíbrio muscular durante a abdução do membro, sobreuso em movimentos repetidos acima da linha do ombro (destaque para esportes de arremesso) e alterações degenerativas próprias do tendão. Dentre os fatores extrínsecos podemos citar o formato e espessura do acrômio, instabilidade glenoumeral, alterações degenerativas acrômioclaviculares, os acromiale, etc ${ }^{4}$.

O principal sintoma descrito na síndrome de impacto é dor em região anterior do ombro, podendo ser acompanhado de fraqueza, que por sua vez pode estar relacionada ao quadro álgico, ou por lesão tendinosa associada. Esta diferenciação é de extrema importância para o clínico, e pode ser feita com a contraprova descrita por Neer com bloqueio anestésico subacromial. A dor de forma habitual tem início insidioso, e raramente ultrapassa o nível do cotovelo, sendo, por outro lado, comum na região lateral do músculo deltóide. Durante a investigação é importante avaliar a relação com movimentos específicos e relação com período do dia ${ }^{1}$.

O exame físico é composto por inspeção, palpação, arco de movimento e testes e manobras especiais.

$\mathrm{Na}$ inspeção, são pesquisadas assimetrias e alterações tróficas a partir do contorno dos grupamentos musculares, ou de sua mensuração direta, quando possível.

Durante a palpação, que deve passar por trapézio, cervical, articulação acrômioclavicular, corredeira bicipital, espaço subacromial e inserção do deltoide, são investigados pontos álgicos, crepitações e tumorações.

A amplitude de movimento deve sempre ser comparativa ao membro contralateral, tanto de forma ativa quanto passiva, esta com auxilio do examinador. Os movimentos básicos a serem testados são a elevação (movimento que ocorre no plano da escápula, em 45 graus entre os planos de flexão e abdução do ombro), a rotação externa e a rotação interna ${ }^{1}$.

São descritos vários testes especiais para patologias subacromiais (impacto), com testes irritativos e funcionais. Testes irritativos são aqueles que reproduzem o impacto de partes moles contra estruturas osteoligamentares. Como exemplo: teste de Neer, teste de Hawkins-Kennedy e teste de Yocum ${ }^{6}$.

1- Teste de Neer: descrito como arco doloroso de Neer, dor subacromial durante elevação passiva do membro entre $70-120^{\circ}$ enquanto o examinador, com a outra mão, mantém a escápula estável;

2- Testes de Hawkins-Kennedy: movimento de rotação interna passiva do membro com flexão de $90^{\circ}$ do ombro. Positivo quando há quadro de dor aguda em região subacromial/deltóidea;

3- Teste de Yocum: com a mão do avaliado apoiada sobre ombro contralateral, procede-se à elevação passiva do cotovelo. Positivo quando há sintomas álgicos em região subacromial ou deltoideana.

Testes funcionais são aqueles descritos para testar a função dos tendões do manguito ou demais estruturas 
do complexo bíceps-labral. Como exemplo, os testes de Jobe, Patte e Napoleão ${ }^{6}$.

1 - Teste de Jobe: força contraresistência para elevação do membro com rotação interna com o membro posicionado no plano da escápula. $\mathrm{O}$ teste é positivo quando há tentativa evidente de força, porém, insuficiência para manutenção da posição do membro;

2 - Teste de Patte: com o cotovelo em flexão de $90^{\circ}$, força contraresistência para rotação externa a partir de uma posição neutra do membro;

3 -Teste de Napoleão: com o cotovelo em flexão de $90^{\circ}$, força contraresistência para aproximar a mão da barriga, em ação de rotação interna.

Fazem parte do arsenal diagnóstico para síndrome de impacto a radiografia, a ultrassonografia e a ressonância nuclear magnética² .

Alguns achados em radiografias podem sugerir o quadro de impacto, dentre eles, a esclerose em tuberosidade maior do úmero na incidência em ânteroposterior, o formato do acrômio visualizado no perfil de escápula e o acromiale visível na incidência em axilar².

A avaliação estrutural do ombro na ultrassonografia inclui: tendões do manguito, tendão do cabo longo do bíceps, articulação acrômio-clavicular, bursa subacromial e labrum posterior ${ }^{2}$.

O exame de Ressonância Nuclear Magnética (RNM) tem excelente acurácia tanto de estruturas do manguito rotador quanto de outras estruturas osteoligamentares relacionadas ${ }^{2}$.

A possibilidade de resultado falso positivo no exame de imagem, ou seja, achados de exames que não estão relacionados com a clínica, especialmente referentes à interpretação de tendinopatia e bursopatia em exames de ultrassonografia do ombro, e o conflito gerado entre procuradores e peritos em decorrência desta interpretação motivaram o presente estudo.

\section{OBJETIVOS}

Avaliar a existência ou não de relação entre os achados de tendinopatia e bursopatia em exames de ultrassonografia do ombro e a conclusão por capacidade ou incapacidade para o trabalho na interpretação dos peritos.

\section{METODO}

\section{Revisão Bibliográfica}

Apesquisa foi realizada com os descritores "bainha rotadora", "ultrassonografia", "avaliação da capacidade de trabalho" e seus respectivos descritores em inglês, rotator cuff, ultrasonography e disability evaluation. A fonte de pesquisa utilizada foi a Medline, o portal da SBOT para o Journal of Bone and Joint (JBJS), a Revista
Brasileira de Ortopedia (RBO) e o Google scholar. A pesquisa foi feita com a associação dos três descritores e posteriormente com os descritores pareados. Foram consultadas apenas referências em inglês e português. Para a descrição conceitual de anatomia, mecanismo de lesão, diagnóstico e tratamento foram utilizados livros textos consagrados na literatura ortopédica.

\section{Roteiro e Aplicação}

Trata-se de um estudo retrospectivo transversal de laudos médicos periciais, todos com consulta pública, do Juizado Especial Federal Previdenciário de Curitiba (JEF) entre as datas de 01.08.2013 e 01.12.2013. Foram avaliados laudos de 3 médicos peritos especialistas na área de ortopedia e traumatologia, todos eles com mais de 5 anos de experiência em avaliação pericial por incapacidade. Um roteiro composto de seis fases rotineiramente aplicado por estes peritos na avaliação das patologias do ombro foi utilizado no presente estudo (Figura.1).

Fase 1: Identificação: Gênero, idade e profissão.

Fase 2: Tempo de evolução: A determinação da evolução dos sintomas é avaliada a partir das informações do periciando em relação ao início das queixas ou do agravamento de sintomas crônicos, e definida como uma data estimada e não exata. $\mathrm{O}$ questionamento utilizado para a investigação é: desde quando o(a) sr(a) acredita que a dor no ombro o incomoda a ponto de lhe prejudicar nas suas atividades habituais?. O tempo deve ser definido em meses e, para fins didáticos, dividido em 3 grupos: grupo 1, aqueles com tempo de evolução inferior a 6 meses; grupo 2, aqueles com evolução entre 6 meses e 18 meses e grupo 3 , aqueles com evolução superior a 18 meses.

Fase 3: Relação dominância/lesão e associações: a dominância e sua relação com o membro comprometido é especificada a partir das siglas: D, para aqueles com membro dominante sendo o comprometido; $\mathrm{N}$, para aqueles com membro comprometido contralateral ao dominante e B, com comprometimento bilateral. As queixas associadas foram definidas pela associação com queixas em coluna lombar, coluna cervical, cotovelo, joelho, mão/punho, pé/tornozelo e fibromialgia.

Fase 4: Exame clínico: Palpação, testes de impacto irritativos e funcionais (força). Na avaliação da palpação utilizada como referência anatômica em avaliação descendente: cervical, trapézio, deltoide lateral, deltoide posterior, deltoide anterior, corredeira bicipital, distal ao cotovelo. De acordo com a interpretação do examinador, foi aplicada a definição de consistente para aqueles em que a dor expressada durante a palpação tinha provável correlação anatômica com as estruturas subacromiais avaliadas, e, inconsistente, para aqueles que a dor expressada tinha pouca correlação 
anatômica com as estruturas estudadas. Para a definição por inconsistente é utilizada a relação de sintomas descritos por Lech, Severo (2003) em especial quanto à dor ultrapassando a linha do cotovelo, ou quanto à presença de dor referida com mesma intensidade em todos os pontos testados (ou seja, o quadro álgico nos pontos relacionados com estruturas subacromiais não predomina sobre os demais, sugerindo inespecificidade dos sintomas).

A partir de exame descrito por Lech, Piluski ${ }^{1}$ (2005), aplicam-se 3 testes irritativos para região subacromial, ou seja, testes provocativos de dor: teste de Neer, teste de Hawkins-Kennedy e teste de Yocum.

Cada caso é graduado como 0 quando os testes resultam negativos, e em 1, 2 ou 3 de acordo com o número de testes positivos. Os testes de força são divididos de acordo com a deficiência do tendão, com aplicação do teste de Jobe para avaliação do tendão supraespinal, Patte para avaliação do tendão infraespinal, e Napoleão para avaliação do tendão subescapular. Cada caso é graduado em 0 para todos os testes negativos, 1 para um teste positivo, 2 para dois testes positivos e 3 quando os três testes são positivos na interpretação do perito.

Fase 5: Exames complementares: Nesta fase, a atenção é voltada para os exames de imagem (complementares) especificamente. Para ultrassonografia, aplica-se a letra B quando o exame de imagem tem descrição isolada de bursopatia, T quando de tendinopatia e TB quando ambos os achados estão presentes. A presença e a associação com exame de RNM também foi anotada.

Fase 6: Determinação da incapacidade/capacidade: Conclusão do perito em referência à capacidade do periciado para o trabalho habitualmente realizado no momento da perícia: capacidade, incapacidade temporária ou incapacidade permanente para o trabalho.

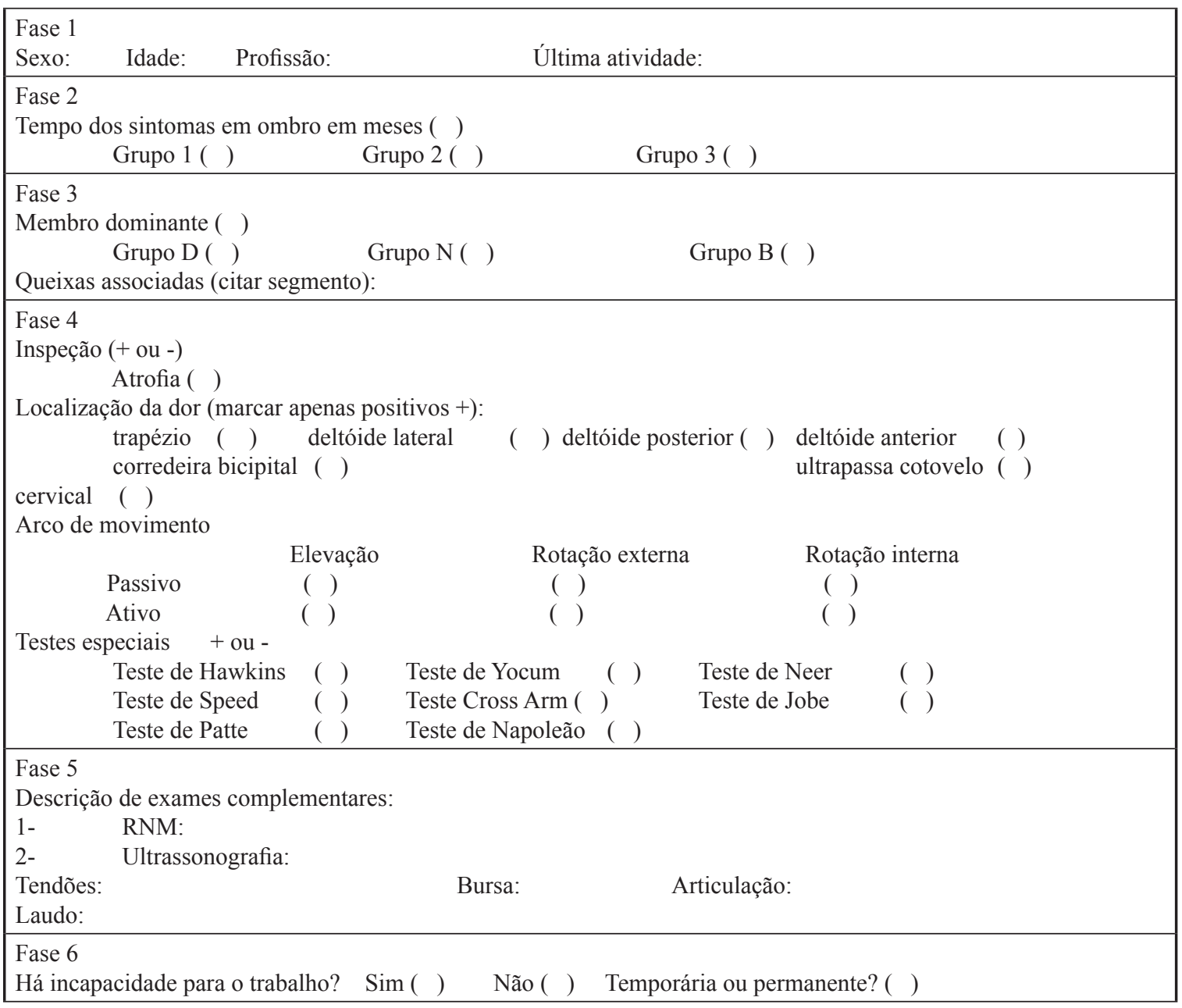

Figura 1 - Roteiro de avaliação pericial das patologias de ombro

No presente estudo foram utilizados como critérios de inclusão, sustentados pelos instrumentos utilizados na pesquisa: dor no ombro como queixa principal de incapacidade, investigação com pelo menos um exame 
de ultrassonografia com no máximo 6 meses de realização da perícia, exame de imagem com laudo descrevendo tendinopatia do tendão supraespinal e/ou bursopatia subacromial. Foram ainda utilizados como critérios de exclusão: cirurgias pregressas, rupturas do tendão de qualquer importância, tendinopatia calcárea, capsulite adesiva, história de luxação prévia, quadro reumatológico comprovado ou aqueles com últimos exames realizados em data mais de 6 meses distante da data da perícia.

Depois de avaliados todos os laudos médicos compreendidos no período delimitado, cumpriram os critérios de inclusão para o estudo apenas 21 deles.

\section{RESULTADOS}

Não foram encontrados artigos específicos referentes ao assunto tratado neste estudo, e, portanto, a base técnica para este trabalho foi constituída por artigos que descreviam os achados de exames de imagem em pacientes assintomáticos, envelhecimento do tendão com suas alterações microestruturais e testes clínicos para síndrome do manguito rotador e suas especificidades.
Dentre os avaliados incluídos no estudo, havia 16 do sexo feminino e 5 do sexo masculino. A idade média foi de 46,4 anos, com variação entre 27 e 64 anos de idade.

Com relação à atividade profissional exercida: no setor industrial e metalurgia foi encontrado o maior número de avaliados, 6 (seis), dos quais 3 (três) estavam vinculados em Carteira de Trabalho e Previdência Social (CTPS) como operadores de máquina e 3 (três) como auxiliares de produção. No setor de limpeza e higienização foram enquadrados 5 (cinco) avaliados, dos quais apenas 1 (um) deles estava vinculado ao Regime Geral de Previdência Social (RGPS) como contribuinte individual, os demais estavam registrados em CTPS como auxiliar de limpeza, servente de limpeza e/ou doméstica. Quatro (4) dos avaliados trabalhavam no setor têxtil, com costura em geral, sendo que 3 (três) destes não possuíam vínculo empregatício comprovado. Ainda, 2 (dois) trabalhavam como auxiliares administrativos, 2 (dois) na área de construção civil, como meio-oficial e pedreiro autônomo respectivamente, 1 (um) em check-out e 1 (um) cozinheiro (Tabela 1).

Tabela 1 - Avaliação da atividade profissional (Fase 1)

\begin{tabular}{lllll}
\hline $\begin{array}{l}\text { Atividade } \\
\text { profissional }\end{array}$ & Indústria e metalurgia & Limpeza e Higienização & Setor têxtil & Outras \\
\hline $\begin{array}{l}\text { Número de } \\
\text { indivíduos }\end{array}$ & $28,6 \%(\mathrm{n}=6)$ & $23,8 \%(\mathrm{n}=5)$ & $19 \%(\mathrm{n}=4)$ & $28,6 \%(\mathrm{n}=6)$ \\
\hline
\end{tabular}

$\mathrm{Na}$ avaliação da evolução dos sintomas, dos 21 avaliados, apenas 1 deles se encontrava no grupo 1 (evolução dos sintomas inferior a 6 meses), 6 no grupo 2 (entre 6 e 18 meses) e 14 no grupo 3 (queixas com mais de 18 meses de evolução) (Tabela 2). A média de tempo de sintomas foi de 30,3 meses, com variação entre 3 e 60 meses.

Tabela 2 - Avaliação do tempo de evolução dos sintomas (Fase 2)

\begin{tabular}{llll}
\hline Tempo de evolução & Grupo 1 & Grupo 2 & Grupo 3 \\
\hline Número de indivíduos & $4,8 \%(\mathrm{n}=1)$ & $28,6 \%(\mathrm{n}=6)$ & $66,7 \%(\mathrm{n}=14)$ \\
\hline
\end{tabular}

Todos os avaliados eram destros. Foram enquadrados 11 avaliados no grupo D (com dominância e sintomas relacionados), 1 avaliado, no grupo $\mathrm{N}$ (dominância contrária aos sintomas) e 9 avaliados no grupo B (sintomas em ambos os ombros).

No que se refere àss queixas álgicas associadas, apenas 4 avaliados não apresentavam outras queixas de dor em segmentos não relacionados aos ombros, e, destes, apenas 2 queixavam-se de dor em apenas um dos ombros. A dor em coluna lombar foi a associação mais freqüente, aparecendo oito vezes (8), seguida de cotovelo (5), cervical e joelho (3 cada uma), mão/punho, tornozelo e fibromialgia (2) (Tabela 3).

Tabela 3 - Avaliação das queixas segmentares associadas ao quadro de ombralgia (Fase 3)

\begin{tabular}{lllll}
\hline Queixas associadas & Coluna lombar & Cotovelo & Coluna cervical & Outras \\
\hline Número de indivíduos & $38,0 \%(\mathrm{n}=8)$ & $23,8 \%(\mathrm{n}=5)$ & $14,3 \%(\mathrm{n}=3)$ & $42,8 \%(\mathrm{n}=9)$ \\
\hline
\end{tabular}


Weiss FP; Zerbini T. Relação entre achados ultrassonográficos de tendinopatia e bursopatia de ombro e incapacidade para o trabalho.

Durante a fase 4 de avaliação, referente ao exame clínico, no item palpação, 7 (sete) dos 21 avaliados descreveram ou expressaram sintomas álgicos que o perito que os avaliou interpretou como condizentes com patologia subacromial. A avaliação foi definida como inconsistente para os outros 14 avaliados.

$\mathrm{Na}$ aplicação dos testes de impacto, 12 (doze) dos 21 casos avaliados não apresentaram nenhum dos testes positivos; 4 (quatro) com 1 teste positivo, 1 (um) com 2 testes positivos, e 4 (quatro) com 3 testes positivos, sendo 2 (dois) destes também com sinais compatíveis com comprometimento de tendão do cabo longo do bíceps (CLB). Apenas 1 dos examinados apresentou testes funcionais positivos para supraespinal $($ Jobe + ) e subescapular (Napoleão +) (Tabela 4).

Tabela 4 - Avaliação dos testes de impacto aplicados (Fase 4)

\begin{tabular}{|c|c|c|c|c|c|c|}
\hline \multirow{2}{*}{$\begin{array}{l}\text { Testes } \\
\text { especiais }\end{array}$} & \multicolumn{3}{|c|}{ Testes de impacto positivo } & \multirow{2}{*}{$\begin{array}{l}\text { Teste para CLB positivo } \\
9,5 \%(n=2)\end{array}$} & \multirow{2}{*}{$\begin{array}{l}\text { Testes funcionais } \\
\text { positivos }\end{array}$} & \multirow{2}{*}{$\begin{array}{l}\text { Ausência de } \\
\text { testes positivos }\end{array}$} \\
\hline & 1 teste & 2 testes & 3 testes & & & \\
\hline & $\begin{array}{l}19,0 \% \\
(n=4)\end{array}$ & $\begin{array}{l}4,8 \% \\
(n=1)\end{array}$ & $\begin{array}{l}19,0 \% \\
(n=4)\end{array}$ & & & \\
\hline
\end{tabular}

Para serem incluídos no estudo, todos os casos deveriam possuir pelo menos um exame de ultrassonografia (US) realizado com antecedência máxima de 6 meses em relação à data da perícia. Destes, 5 deles também apresentavam exame de RNM dentro dos limites de tempo pré-definidos. Os exames de US e RNM foram concordantes em 02 casos. As 03 discordâncias foram devidas à associação do comprometimento de outras estruturas (bursopatia, tendinopatia de SE e tendinopatia de SB) em exame de RNM, mas nenhuma destas estava relacionada à constatação de lesão do tendão que não tivesse sido diagnosticada no exame de ultrassonografia. Dos exames de ultrassonografia avaliados, 10 foram laudados como tendinopatia e bursopatia, 7 apenas como tendinopatia e 4 apenas como bursopatia.

Dos 21 avaliados, 9 (42,9\%) mostravam algum sinal de impacto subacromial, enquanto $12(57,1 \%)$ não possuíam nenhum sinal clinico compatível com impacto na interpretação dos examinadores.

De todos os casos avaliados, os examinadores concluíram por incapacidade em 3 casos (14,3\%), sendo todos com definição por incapacidade temporária e nenhum permanente. Nas outras 18 avaliações $(85,7 \%)$ os médicos examinadores concluíram pela capacidade para o trabalho habitualmente desenvolvido (Figura 2).

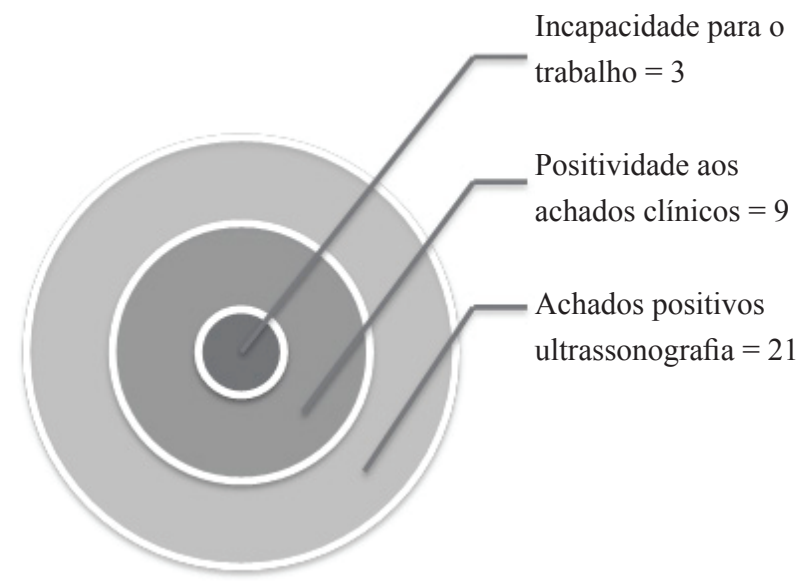

Figura 2 - Relação entre achados positivos em exames de imagem, positividade aos testes clínicos e incapacidade para o trabalho.

\section{DISCUSSÃO}

Conforme descrito por $\operatorname{Neer}^{5}$ (1983), a síndrome de impacto se desenvolve em três estágios: o primeiro estágio, com edema e hemorragia, em pacientes com idade típica inferior aos 25 anos; o segundo estágio, de ocorrência prevalente entre 25 e 40 anos, com fibrose e tendinite, e um terceiro estágio, habitualmente em pacientes com mais de 40 anos, no qual são encontrados esporões ósseos e ruptura do tendão, e que habitualmente apresentam incapacidade progressiva. No presente estudo foram incluídos apenas casos que pela avaliação 
ultrassonográfica estivessem em estágio 1 ou 2 da classificação de Neer, ou seja, sem ruptura do tendão. Nesta amostra a média de idade encontrada foi de 46,4 anos, variando de 27 a 64 anos.

Sob o aspecto médico-legal, o tempo de evolução dos sintomas e a forma de sua progressão trazem informações valiosas para o perito com respeito à compatibilidade entre as informações do avaliado com aquela descrita na literatura médica para as doenças do manguito rotador.

Azar $(2007)^{2}$ cita que, em casos de impacto do ombro, após 3 a 4 meses de tratamento clínico sem sucesso, há indicação de tratamento cirúrgico com acromioplastia. Esta, por sua vez, obteve sucesso em aproximadamente $85 \%$ dos casos, sendo as falhas atribuídas a um diagnóstico incorreto, falha técnica e outras complicações. Lech, Severo ${ }^{1}$ (2003) citam Neer em sua conduta prática de aguardar um período entre $3 \mathrm{e}$ 6 meses com tratamento conservador antes de indicar o tratamento cirúrgico.

Bigliani e Levine ${ }^{4}$ (1997), em revisão, citam estudos referentes ao tratamento conservador da síndrome de impacto que variam em tempo entre 12-18 meses. Descrevem ainda que o tempo de 6 meses é um tempo estimado adequado para a indicação do tratamento cirúrgico na falha do tratamento clínico. Morrison et al ${ }^{7}$ (1997), em avaliação de 636 ombros com diagnóstico de impacto subacromial submetidos a tratamento conservador, com seguimento de 27 meses, obtiveram resultados satisfatórios em 413 (67\%). Também concordam com os $67 \%$ de bons resultados naqueles pacientes com mais de 6 meses de sintomas. Destes, $18 \%$ tiveram recidivas posteriores do quadro clínico. $\mathrm{O}$ resultado do sucesso do tratamento conservador com base nos dados deste autor também foi relacionado diretamente com o formato do acrômio, com $91 \%$ de melhora em casos de acrômio tipo I, contra $68 \%$ no tipo II e $64 \%$ no tipo III.

No presente estudo o tempo de evolução dos sintomas teve variação de 3 meses a 60 meses, com média de 30,4 meses. Esta média é superior ao tempo habitual para resolução dos sintomas de impacto do ombro em relação à literatura. Esta diferença pode ser atribuída à dificuldade de acesso ao tratamento ou à efetiva falta de busca ativa pelo tratamento. Cabe ao médico perito fazer tal diferenciação no momento da avaliação, especialmente pela comprovação documental presente nos autos, apresentação de atestados, comprovantes de fisioterapia e descrições de prontuários médicos. Tal avaliação pode ser um dos fatores de interferência na decisão do perito sobre a capacidade ou incapacidade para o trabalho daquele indivíduo.

Outro aspecto relevante na avaliação pericial é o diagnóstico equivocado por parte dos médicos assistentes em relação às patologias do ombro de seus assistidos, o que acaba trazendo uma dificuldade a mais para o perito no momento da avaliação pericial.

O número de estruturas envolvidas no complexo mecanismo de movimento do ombro o torna susceptível a lesões e torna muito difícil o raciocínio diagnóstico do clínico. Ao longo dos anos parece haver um condicionamento dos clínicos a atribuir as queixas de dor em região anterior do ombro à síndrome de impacto, quando na verdade inúmeros diagnósticos diferenciais devem ser levados em conta nesta situação, como instabilidade ligamentar, osteoartrose, capsulite adesiva, tendinite calcárea, radiculopatia cervical e neuropatias ${ }^{4}$.

O presente estudo detectou ainda que, dentre os indivíduos que se enquadravam nos critérios de inclusão, apenas 2 dos 21 avaliados queixavam-se de dor apenas em um ombro, e 19 apresentavam queixa em mais de uma articulação ou segmento anatômico. Estes achados podem sugerir um diagnóstico sindrômico distinto da síndrome de impacto, coincidência sobre a concomitância dos sintomas ou, ainda, a possibilidade de sobrevalorização das queixas no momento da avaliação.

O exame físico, com seus testes especiais, é de suma importância para o diagnóstico da lesão e para caracterização do grau de limitação que esta gera. Estudo de Silva et $\mathrm{al}^{8}$ (2008) comparou a relação entre os achados do exame clínico com os achados de exame de RNM, e encontrou sensibilidade relativamente boa dos testes clínicos aplicados (acima de 58\%), porém, com baixa especificidade, com variação entre 10-60\%,

Outro estudo, este de Jia et al $^{9}$ (2009), concluiu que tanto o teste de Neer quanto teste de HawkinsKennedy têm boa sensibilidade para detecção de quadro de tendinose de supraespinal e ruptura parcial do manguito, mas ambos com baixa especificidade.

O diagnóstico complementar da tendinopatia do ombro pode ser auxiliado por radiografias, ultrassonografias e exames de ressonância nuclear magnética. Em avaliações de patologias do ombro a ultrassonografia (US) é hoje o exame de maior prevalência nas perícias no Juizado Especial Federal Previdenciário de Curitiba. A US é um exame de acurácia próxima ao exame de RNM para a avaliação de rupturas tendinosas e também para os achados cirúrgicos (Moosmayer, Smith, $2005)^{10}$, o que faz que sua utilização seja feita em larga escala, especialmente pelo baixo custo e possibilidade de imagens dinâmicas. Por outro lado, a ultrassonografia é um exame examinador-dependente, e pode ter sua acurácia alterada, por exemplo, de um centro diagnóstico para outro. ${ }^{11}$

Para a correta interpretação dos exames de imagem, inicialmente devemos compreender que o nível de hidratação do tendão. Modificações de sua composição e estrutura certamente ocorrem com o envelhecimento, e estas possivelmente serão detectadas nos exames de imagem. 
As doenças do manguito rotador envolvem um espectro de condições específicas, desde uma tendinopatia incipiente até a ruptura completa do tendão. A homeostase do tendão depende da relação entre a variabilidade celular, componentes da matriz, enzimas, citocinas, fatores de crescimento e proteínas ${ }^{12}$. Uma alta prevalência e distribuição difusa de alterações degenerativas foi encontrada em avaliações histológicas do manguito rotador, incluindo afilamento e desorganização das fibras de colágeno, degeneração mixoide, degeneração hialina, metaplasia condral, calcificação, proliferação vascular, e infiltração gordurosa. Todas as alterações, com exceção da proliferação vascular e infiltração gordurosa, foram encontradas em maior grau nas camadas médias e profundas do tendão em detrimento à camada superficial ${ }^{13}$.

Alterações próprias do tendão detectadas em exames de imagem comumente ficam definidas de forma genérica como tendinopatias, visto que a diferenciação de tendinose e tendinite por meio de exames de imagem não é uma tarefa fácil. Por critérios histopatológicos a tendinose pode ser definida como a alteração degenerativa das fibras do tendão com nenhum ou pouco processo inflamatório, enquanto a tendinite tem como principal componente a inflamação. O que resta amplamente aceito na literatura é que a tendinopatia é uma falha do mecanismo de reparo do tendão ${ }^{14}$. O mecanismo da evolução da tendinopatia pode ser simulado pela hipersolicitação, mas também pela hipossolicitação, pois os achados anatomopatológicos são semelhantes.

Neviaster et al $(2012)^{15}$, em artigo de revisão a respeito dos mecanismos de fadiga do tendão, concluíram que alterações moleculares, mecânicas e estruturais do tendão continuam com sua origem pouco definida, e que tais alterações não podem ser atribuídas isoladamente à fatores extrínsecos ou intrínsecos, mas sim definida como multifatorial. Acrescenta ainda que as lesões cumulativas do tendão têm influência do uso repetido (ciclo-fadiga) e a idade.

Em exames de ultrassonografia utiliza-se como critério para definir tendinopatia (quadros de tendinose e/ou tendinite): a redução difusa da ecogenicidade do tendão, áreas hipoecóicas mal-definidas acompanhando o sentido das fibras, associadas ou não ao aumento de diâmetro do tendão, calcificações distróficas caracterizadas por focos hiperecóicos, acompanhados ou não por sombra acústica posterior. Define ainda ruptura parcial em achados de lesão hipoecogênica e/ou presença de descontinuidade das fibras ou lesão mista caracterizada por lesão hiperecóica com halo hipoecóico; são sinais de rupturas totais a não oservação do tendão ou descontinuidade do tendão. Sinais indiretos de ruptura também devem ser procurados, como coleções líquidas no espaço subacromial ou acrômio-clavicular, ausência focal do tendão, espessamento da bursa, irregularidade da face óssea da articulação, sinal da interface da cartilagem, contorno côncavo da gordura subdeltoidea ${ }^{16}$. Conclui ainda o autor que por ser um método de baixo custo e com boa sensibilidade em mãos experientes, é um bom método diagnóstico para investigação inicial em quadros de síndrome de impacto.

As lesões do manguito rotador habitualmente são consequência do envelhecimento do tendão, e frequentemente, apesar de presentes em exames de imagem, estas não repercutem em sintomas. $\mathrm{O}$ tratamento deve ser indicado pelos sintomas e sinais apresentados e não pelos achados de exame. Para ressaltar a necessidade de valorização criteriosa dos exames complementares, estudo de Milgrom et $\mathrm{al}^{17}$ (1995) encontrou em exames de ultrassonografia lesão parcial ou completa de manguito rotador em $50 \%$ dos avaliados assintomáticos na sétima década de vida, e $80 \%$ na oitava década de vida. Dentre os achados em avaliados assintomáticos, o autor ainda encontrou lesão parcial de manguito em $5 \%$ na $3^{\text {a }}$ década e $11 \%$ na $4^{\text {a }}$ década. Neste estudo não foi encontrada relação entre as lesões com esforços do ombro (não estudada população esportista) ou com a dominância da mão.

Adiscordância entre achados de exames de imagem e clínica apresentada é também divergente na literatura médica. $\quad$ Estudo publicado por Naredo et $\mathrm{al}^{18}$ (2002), definiu que a avalição clínica, relacionada aos testes específicos de impacto tem baixa acurácia em relação aos achados de exames de ultrassonografia, e atribuiu seus resultados à baixa correlação entre os testes clínicos específicos com alterações anatômicas. Neste estudo, todos os pacientes com lesão completa dos tendões do manguito, tenossinovite do supraespinal ou bursite subacromial apresentavam sinais clínicos compatíveis. Porém, quando achados ultrassonográficos mostravam sinais de impacto do supraespinal, lesão parcial do tendão ou sinais de artrose do ombro, o autor encontrou muitos pacientes sem sinais clínicos correspondentes.

Estudo de Girish et $\mathrm{al}^{11}$ (2011) avaliou 51 homens, assintomáticos, média de 56 anos, e apontou alterações ultrassonográficas em $96 \%$ dos indivíduos. Dentre os principais achados: o espessamento da bursa subacromial/ subdeltoidea, artrose acrômio-clavicular, e tendinose do supraespinal. Neste mesmo estudo o autor pôde concluir que $39 \%$ dos exames mostravam tendinose do supraespinal, $22 \%$ ruptura parcial do tendão supraespinal e $10 \%$ ruptura total. Dentre as bursopatias, os resultados foram ainda mais significativos: $79 \%$ dos indivíduos com espessamento da bursa subacromial/subdeltoidea. Ainda, 65\% com sinais de artrose da articulação acrômioclavicular. Com base neste trabalho, o autor recomenda que os achados de exame sejam interpretados com grande critério clínico para definir a real causa dos sintomas em patologias do ombro.

Em nosso estudo, dos 21 indivíduos avaliados com diagnóstico de síndrome de impacto em ombro 
pelos médicos assistentes, e achados de exames de ultrassonografia que indicavam tendinopatia e/ou bursopatia, os peritos avaliadores detectaram achados clínicos compatíveis com patologia subacromial no exame de palpação em 7 (33\%), havendo sinais sugestivos de impacto subacromial pelas manobras irritativas em $9(42,8 \%)$. Destes, quatro com 3 manobras positivas, um com 2 manobras positivas e quatro com 1 manobra positiva. Porém, os peritos concluíram por incapacidade para o trabalho apenas em 3 dos 21 examinados (14,3\%). Todos os examinados nos quais não houve detecção de sinais de impacto nos testes irritativos foram considerados capazes para o trabalho. Este dado indica que os peritos consideraram, dentro desta amostragem, que a ausência de relação entre os testes físicos e os exames de imagem é um elemento suficiente para definir a ausência de incapacidade para o trabalho. $\mathrm{Ou}$ seja, o primeiro critério fundamental utilizado para caracterização de incapacidade por tendinopatia/ bursopatia de ombro é a presença de sinais clínicos característicos da lesão e da doença, o que concorda com a bibliografia.

Por outro lado, a presença dos sinais de impacto não foi elemento determinante de incapacidade para o trabalho pela interpretação dos peritos, visto que 6 dos 9 indivíduos do estudo que apresentavam sinais de impacto não foram considerados incapazes para suas respectivas atividades profissionais pelos peritos.

Devemos compreender que este estudo foi realizado em período curto (quatro meses), permitindo a inclusão de apenas 21 casos, amostra considerada pequena para que seus resultados possam ser aplicados para a população geral.

\section{CONCLUSÃO}

Concluiu-se pelo presente estudo, frente à amostragem obtida, que: A existência de achados de tendinopatia e/ou bursopatia em exame de ultrassonografia do ombro não teve relação com a conclusão dos peritos pela incapacidade laboral para o grupo avaliado, pois apenas $14,3 \%$ daqueles indivíduos avaliados foram considerados incapazes para o trabalho. Dentre os avaliados com exame de ultrassonografia que indicava tendinopatia e/ou bursopatia, os peritos não encontraram sinais clínicos compatíveis em mais da metade dos casos estudados, concluindo em todos estes casos pela capacidade para o trabalho.

\section{CONSIDERAÇÕES FINAIS}

As limitações do presente estudo estão relacionadas às inúmeras variáveis a serem consideradas para o raciocínio e conclusão do perito, como: a profissão executada pelo periciado e sua profissiografia, a relação de vínculo empregatício ou não, o tempo de evolução dos sintomas e a compatibilidade entre a descrição do quadro clínico pelo periciado com aquela descrita na literatura. Tais variáveis, apesar de inicialmente incluídas no método do estudo, não puderam ser bem compreendidas pelo pequeno número da amostragem, sendo traduzidas em hipóteses a serem estudadas em futuros projetos.

Weiss FP; Zerbini T. Relationship between tendinopathy and bursopathy findings in ultrasonography of the shoulder and work disability. Saúde, Ética \& Justiça. 2013;18(2):157-66.

\begin{abstract}
S: Tendinopathy and bursopathy findings are common in ultrasonography of shoulder, but frequently there are no clinical symptoms. Consequently, these findings may be not understood by experts as disease or lesions. This causes a lot of objections by prosecutors and judges, raising doubts about the quality of the expert report. The present study aims to assess the interpretation of the medical experts regarding the relationship between work disability and the tendinopathy or bursopathy as was shown by the ultrasound of the shoulders. For this purpose, expert reports were selected from the Juizado Especial Federal Previdenciário de Curitiba (the Special Federal Court for Social Security in Curitiba) in which the main complaint was shoulder pain and the ultrasonography findings were according to the study goals. The results were evaluated considering the clinical signs, the ultrasonography findings and the expert conclusions about work disability. Among the 21 individuals included in the study, there was correspondence between alterations found in exams and positive results of clinical tests in just $42.8 \%$ of cases. Of these, only $33 \%$ were defined as unable to work. This study concluded that only $14.3 \%$ of individuals with tendinopathy and bursopathy findings were considered unable to work, which indicates that isolated findings of shoulder ultrasonography are not enough for the assessment of technical ability to work.
\end{abstract}

KEYWORDS: Rotator cuff; Ultrasonography; Employee performance appraisal.

\title{
REFERÊNCIAS
}

1. Lech O, Severo A. Ombro e cotovelo. In: Hebert S, Xavier R. Ortopedia e traumatologia, princípios e prática. $3^{\mathrm{a}} \mathrm{ed}$. Porto Alegre: Artmed; 2003. p185-213.

2. Azar FM. Lesões do Ombro e Cotovelo. In: Canale ST, editor. Cirurgia ortopédica de Campbell. Trad de Nelson Gomes de Oliveira. Barueri. 10 $0^{\mathrm{a}}$ Ed. São Paulo: Manole; 2007. p. 2341-2.

3. Moore KL, Daley AF. Anatomia orientada para a clínica. 
Weiss FP; Zerbini T. Relação entre achados ultrassonográficos de tendinopatia e bursopatia de ombro e incapacidade para o trabalho.

Trad. de Alexandre Lins Werneck e Wilma Lins Werneck. $4^{\mathrm{a}}$ ed. Rio de Janeiro: Guanabara Koogan; 2001. 620p.

4. Bigliani LU, Levine WN. Current concepts review: subacromial impingement syndrome. J Bone Joint Surg Am. 1997; 79A(12):1854-68.

5. Neer CS. Impingement lesions. Clin Orthop Relat Res. 1983; 173:70-7.

6. Lech O, Piluski P. In Franco JS. editor. Ombro e Cotovelo: Sociedade Brasileira de Cirurgia de Ombro e Cotovelo. Rio de Janeiro; Revinter; 2005. p51-76.

7. Morrison DS, Frogameni AD, Woodworth P. Non-operative treatment of subacromial impingement syndrome. J Bone Joint Surg Am. 1997; 79(5): 732-7.

8. Silva L, Andréu JL, Muñoz P, Pastrana M, Millán I, Sanz $\mathrm{J}$, et al.. Accuracy of physical examination in subacromial impingement syndrome. Rheumatology (Oxford). 2008; 47(5):679-83. DOI: http://dx.doi.org/10.1093/ rheumatology/ken101

9. JIA X, Petersen SA, Khosravi AH, Almareddi V, Pannirselvam V, McFarland EG. Examination of the shoulder: the past, the present and the future. J Bone Joint Surg Am. 2009; 91(Suppl6):10-8. DOI: http://dx.doi. org/10.2106/JBJS.I.00534

10. Moosmayer S, Smith HS. Diagnostic ultrasound of the shoulder- a method for experts only? Results from an orthopedic surgeon with relative inexperience compared to operative findings. Acta Orthop. 2005; 76(4):503-8. DOI: http://dx.doi.org/10.3109/17453674.2010.483993

11. Girish G, Lobo LG, Jacobson JA, Moraq Y, Miller B, Jamadar DA. Ultrasound of the shoulder: asymptomatic findings in men. AJR Am J Roentgenol. 2011; 197(4):W713-
9. DOI: http://dx.doi.org/10.2214/AJR.11.6971

12. Dean BJ, Franklin SL, Carr AJ. A systematic review of the histological and molecular changes in rotator cuff disease. Bone Joint Res. 2012; 1(7):158-66. DOI: http://dx.doi. org/10.1302/2046-3758.17.2000115

13. Hashimoto T, Nobuhara K, Hamada T. Pathologic evidence of degeneration as a primary cause of rotator cuff tear. Clin Orthop Relat Res. 2003; (415):111-20. DOI: http://dx.doi. org/10.1097/01.blo.0000092974.12414.22

14. Mafulli N, Longo UG, Denaro V. Novel approaches for the management of tendinopathy. J Bone Joint Surg Am. 2010; 92(15): 2604-13. DOI: http://dx.doi.org/10.2106/ JBJS.I.01744

15. Neviaser A, Andarawis-Puri N, Flatow E. Basic mechanisms of tendon fatigue damage. J Shoulder Elbow Surg. 2012; 21(2): 158-63. DOI: http://dx.doi.org/101016/j/ jse.2011.11.014.

16. Zorzetto AA, Urban LABD, Liu CB, Prevedello L, Zapparoli M, Vitola MLA, et al. A ecografia no diagnostico das lesões músculo-tendinosas do ombro. Radiol Bras. 2003; 36(4):237-242. DOI: http://dx.doi.org/10.1590/ S0100-39842003000400010

17. Milgrom C, Schaffler M, Gilbert S, van Holsbeeck M. Rotator-cuff changes in asymptomatic adults: the effect of age, hand dominance and gender. J Bone Joint Surg Br. 1995; 77(2): 296-8.

18. Naredo E, Aguado P, De Miguel E, Uson J, Mayordomo L, Gijon-Baños J, et al. Painful shoulder: comparison of physical examination and ultrasonographic findings. Ann Rheum Dis. 2002; 61:132-6. DOI: http://dx.doi. org/10.1136/ard.61.2.132 